\title{
Ultraviolet emission line imaging of planetary nebulae with GALEX
}

\author{
Luciana Bianchi ${ }^{1}$, Arturo Manchado ${ }^{2}$ and Karl Forster ${ }^{3}$ \\ ${ }^{1}$ Johns Hopkins University, Dept. of Physics and Astronomy \\ 3400 N. Charles St. Baltimore, MD 21218,USA \\ email: bianchi@pha.jhu.edu \\ ${ }^{2}$ Instituto de Astrofísica de Canarias, Tenerife, Spain \\ ${ }^{3}$ California Institute of Technology, Pasadena, USA
}

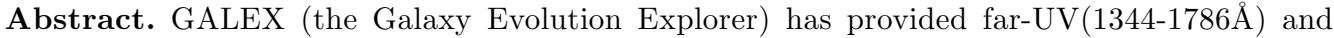
near-UV(1771-2831 $\AA$ ) imaging of several Planetary Nebulae (e.g., Bianchi et al. 2008, Bianchi 2012), with flux limits $\sim 27.5 \mathrm{mag} / \mathrm{sq}$.arcsec for objects in the Medium-deph Imaging Survey (MIS). PNe images in the GALEX broad-band UV filters include flux from both nebular line and continuum emission. We use the GALEX grism observing mode to obtain slitless spectral imaging of a sample of PNe with diameters $>1^{\prime}$, in the near-UV. We show the first data from this program. The grism produces $2 \mathrm{D}$ images of the prominent UV nebular emission lines, when such lines dominate the flux. Combined with monochromatic images of diagnostic lines in the optical domain, such data help detect and interpret ionization and shock fronts, especially in faint nebular regions.
\end{abstract}

Keywords. atlases, planetary nebulae: general, ultraviolet: ISM, ultraviolet: stars, stars: AGB and post-AGB

We are using the GALEX grism observing mode for unraveling the nature of the UV emission seen in GALEX direct images of PNe. In slitless spectroscopy, flux from continuum-emitting extended sources is confused, but for discrete emission lines 2D images are produced along the dispersion direction. For large objects, these overlap in the NUV range where many lines may fall at nearby $\lambda$ 's, due the $\mathrm{R} \sim 100$ grism resolution.
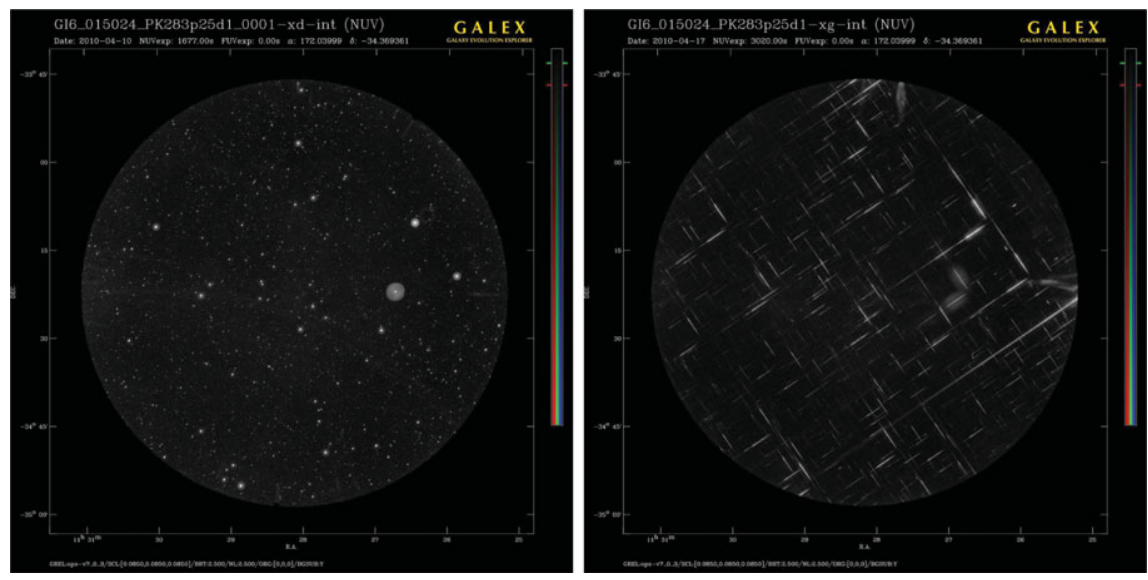

Figure 1. Left: GALEX NUV image of PK283+25 1. Right: co-add of two single NUV grism images taken with different orientations to minimize loss of spectral portions from overlap of nearby sources in the final extracted spectrum. Note two bright stars NW of the PN: their apparent "halo" is the outer NUV psf tail, showing in these saturated objects. The field of view has a $1.2^{\circ}$ diameter 


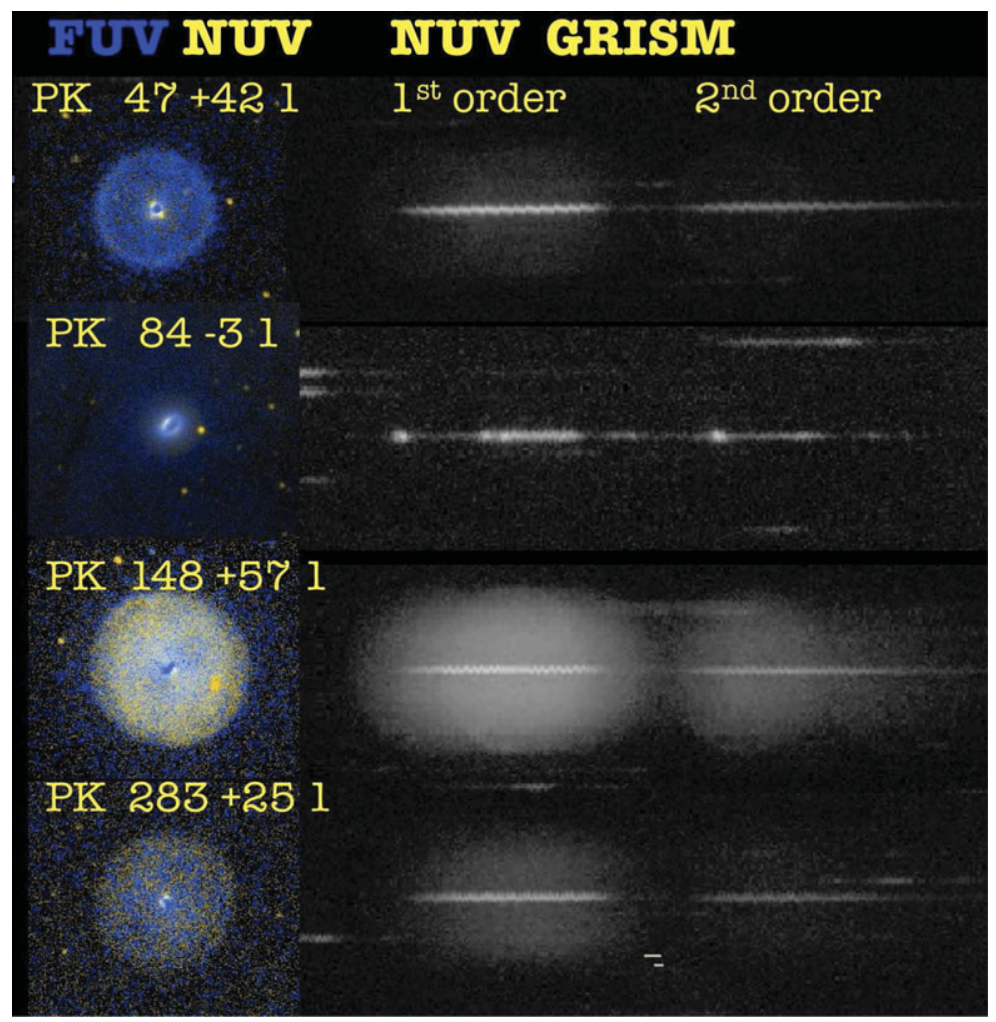

Figure 2. GALEX color-composite imaging (left) of 4 PNe (blue: FUV, yellow: NUV), for which NUV grism data were taken (right). Images are cut to the same size. PK84 -3 1 (NGC 7027) is in an area of blue diffuse cirrus. The grism data show the CSPN continuum, and PN spectral images in several emission lines (often overlapping), with nebular continuum emission possibly in PK 283+25 1 (K 1-22). The NUV range includes CIII] $\lambda 1909$ at its short-wavelength end.

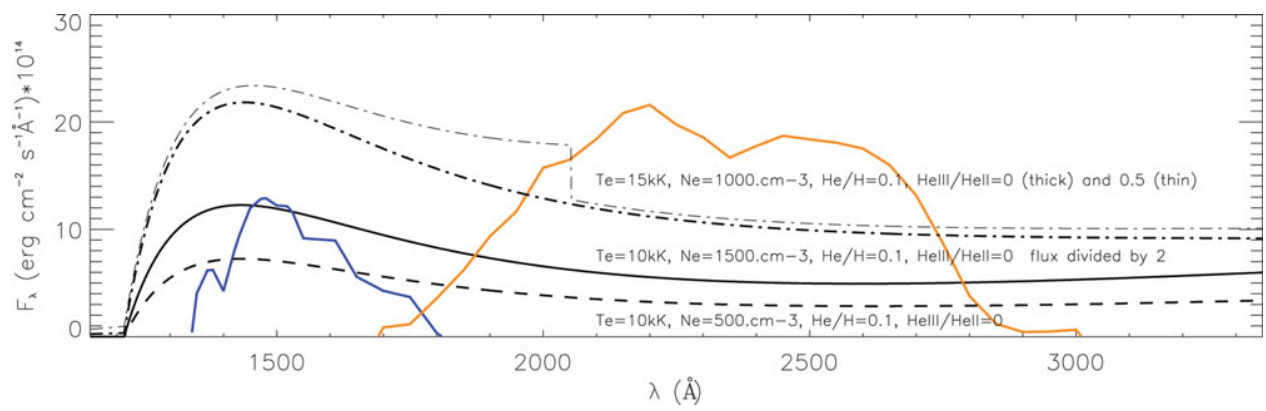

Figure 3. Nebular continuum emission computed for a range of electron temperature $\left(\mathrm{T}_{e}\right)$ and density $\left(\mathrm{N}_{e}\right)$, He abundance and ionization, for a $\mathrm{PN}$ with radius $=60^{\prime \prime}$ at $10 \mathrm{pc}$. FUV, NUV transmission curves of GALEX imaging are overplotted, on arbitrary scale.

The FUV detector stopped functioning after this program was approved, therefore we lost the capability of imaging CIV.

Acknowledgments Support was provided by NASA grant NNX11AI58G (Galex Gi6-15) References

Bianchi, L., 2012, these proceedings

Bianchi, L. \& Herald, J. 2008, in A. I. Gomez de Castro \& N. Brosh (eds.), Space Astronomy: The UV Window to the Universe (Springer) 